\title{
Free oscillations of thin-walled bimetallic pipelines of large diameter on an elastic foundation
}

\author{
Vladimir Sokolov ${ }^{1}$, Igor Razov ${ }^{1 *}$ and Evgeniy Koynov ${ }^{1}$ \\ ${ }^{1}$ Industrial University of Tyumen, 625001, Volodarskogo str. 38, Tyumen, Russia
}

\begin{abstract}
In the paper, solutions for a thin-walled bimetallic pipeline are obtained. The frequencies of free oscillations are investigated, taking into account the internal working pressure, the longitudinal compressive force, and the elastic foundation. The solutions were obtained on the basis of a geometrically nonlinear version of the semi-momentum theory of cylindrical shells of the middle bend. The proposed calculations can be used in the nuclear power industry, aviation, and petrochemical industry.
\end{abstract}

\section{Introduction}

Double-layer steels belonging to the category of bimetals, having a base layer of structural steel and plating of corrosion-resistant, are a unique material combining the required mechanical properties and the necessary corrosion resistance. The main indicators of the quality of double-layer rolling, determining its adaptability in the manufacture of equipment and the operational properties of the product, are the continuity and strength of the layer interconnection, as well as the special properties of the cover layer (corrosion resistance, wear resistance, etc.). The increasing demand for high-performance equipment for oil and gas processing and chemical industries operating in the most extreme conditions (high and low temperatures, pressure, corrosive environments, cyclic loading) necessitates the use of reliable bimetals ensuring compliance with Rostekhnadzor's requirements for fire and explosion hazard facilities and safe operation of equipment for many years. Bimetallic pipelines have several advantages over single-layered steel, especially in terms of corrosion resistance. Such pipelines, as well as single-layer steel, can work under hazardous dynamic conditions. Therefore, this issue is interesting and relevant for the study [1-8].

\section{Materials and methods}

Bimetallic pipelines consist of two layers of metals. Most often the main layer is made of strong steels 10, 20, 09Г2C, 12XM etc., covering of chromium steels $08 \mathrm{X} 13,08 \mathrm{X} 14$ ФБ and chromium-nickel steels $08 \mathrm{X} 18 \mathrm{H} 10,08 \mathrm{X} 18 \mathrm{H} 10 \mathrm{~B}$ etc. The covering layer can be positioned both inside the pipe and/or outside. The location of this layer depends on the field of application of the pipelines, the environmental conditions, and the required mechanical

\footnotetext{
*Corresponding author: RazovIO@mail.ru
} 
characteristics. The main production methods for obtaining bimetallic composites are the following:

1. Cold and hot rolling.

2. Casting.

3. Welding by explosion.

4. Vacuum-diffusion welding.

In addition, there are other methods, such as pressing and drawing, soldering, surfacing, electroslag welding, cold welding, diffusion welding, ultrasonic welding, friction welding. However, these methods are used much less often. Rolling is used for the production of bimetallic sheets, pipes, profiles, rods. The technology of hot rolling of several layers of metal includes preparation (cleaning, etching, washing, degreasing) and assembling the components of the package, preheating and rolling, cutting, heat treatment, straightening, and finishing. To prevent the oxidation of rolled layers, various methods are used - coatings applied electrolytically, by surfacing or spraying, layers of foil and pyrophoric substances. Magnesium carbonate, metal carbides, chlorides, iodides, and metal fluorides are used as reducing substances. For rolling $16 \Gamma \mathrm{C}-12 \mathrm{X} 18 \mathrm{H} 10 \mathrm{~T}$, a mixture of carbon black with liquid hydrocarbons is used in a ratio of 1:4. To ensure better adhesion, intermediate layers (precladding) are also used. Schemes of preliminary laying of bimetallic billets in bags are shown in Figure 1.

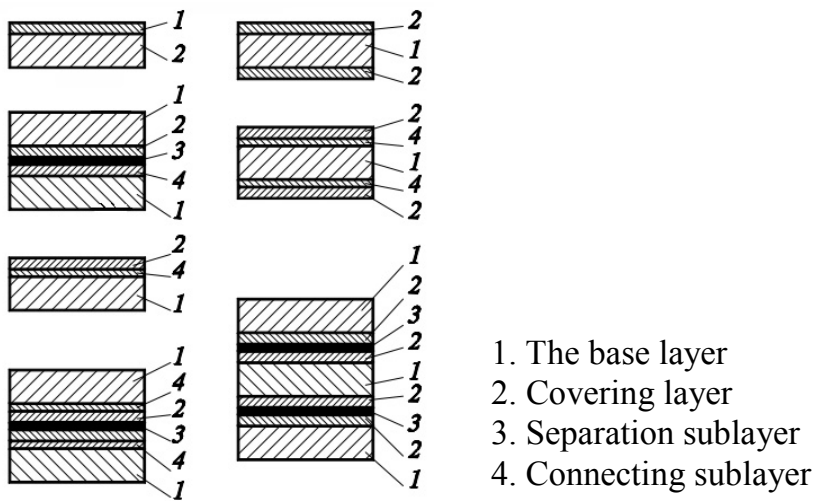

Fig. 1. Schemes of preliminary laying of bimetallic billets.

Until recently, the disadvantage of bimetallic large diameter pipelines was the inability to provide the same properties of the base metal and welded joint, which reduced the operational reliability of the pipes due to insufficient resistance to brittle fracture of the weld and the termination of the ends in the volume heat treatment of the pipes [1]. In addition, field and complex laboratory tests of pipes showed that corrosion is most often observed in the place of welded pipelines, while the overall corrosion of the inner surface of the pipe does not exceed $0.01 \mathrm{~mm} /$ year, and local corrosion is not observed. In this regard, the issue of protection of the welded seam after removal of the bead by welding a narrow strip of the welded seam and by arc welding with an automatic tracking system of the process in the place of absence of the clad layer is topical [2]. Nevertheless, at the present time, there is a technique for producing long length multilayer bimetallic pipes of large diameter for transportation of hydrocarbons. However, there are also difficulties in the use of these materials. In metals, due to the difference in materials by mechanical and thermophysical properties, the distribution of stresses across layers can also be successful or unsuccessful. Therefore, before offering such pipes for any technical devices, it is necessary to study the general laws of the formation of stress fields in multilayer pipes under different thermal stress loading regimes. In the works of K.M. Gumerov, the issue related to the stress fields of 
bimetallic pipelines [3] is studied in detail. In particular, the characteristic cases of operation of pipelines are considered:

- The double-layer tube does not have internal pressure in a cyclically changing uniform temperature field (without gradients);

- The double-layer pipe is under internal pressure at a constant temperature $t_{0}$;

- The double-layer pipe is under the influence of internal pressure and a cyclically changing uniform temperature field;

- The double-layer pipe is under the influence of internal pressure in a non-uniform and cyclically changing temperature field.

In addition, when using these pipes, an economic question arises, since this type of material is an order of magnitude higher than standard steel pipes. However, due to savings on insulating materials and increased service life of these pipelines in corrosive environments, the use of this type of material is economically viable [4].

\section{Results}

Based on the geometrically nonlinear version of semi-momentum theory of cylindrical shells $[5,6,7]$ in the deformed stage, the motion equation is:

$$
\begin{gathered}
\frac{\partial^{2} T_{1}}{\partial \xi^{2}}+\frac{\partial}{\partial \xi}\left(\tau \frac{\partial M_{2}}{\partial \theta}\right)-\frac{\partial^{2}}{\partial \theta^{2}}\left(\frac{R_{2}^{*}}{R_{1}^{*}} T_{1}\right)-\frac{1}{R^{2}} \frac{\partial^{2}}{\partial \theta^{2}}\left(R_{2}^{*} \frac{\partial^{2} M_{2}}{\partial \theta^{2}}\right)- \\
-\frac{\partial}{\partial \theta}\left(\frac{1}{R_{2}^{*}} \frac{\partial M_{2}}{\partial \theta}\right)+R \frac{\partial X_{1}}{\partial \xi}-R \frac{\partial X_{2}}{\partial \theta}-\frac{\partial^{2}}{\partial \theta^{2}}\left(R_{2}^{*} X_{3}\right)=0,
\end{gathered},
$$

where $X_{1}=-R_{0} h \rho_{n p} \frac{\partial^{2} u}{\partial t^{2}}, X_{2}=-R_{0} h \rho \frac{\partial^{2} v}{\partial t^{2}}, X_{3}=-R_{0} h \rho \frac{\partial^{2} w}{\partial t^{2}}+p_{0}-\Psi(\theta) w(\xi, \theta, t)$

$M_{1}$ and $M_{2}$ - bending moments in the longitudinal and cross (circular) directions: $T_{1}$ and $T_{2}$ - longitudinal and circumferential tangential forces; $\aleph_{2}$ - change the coordinate lines of curvature $\theta$ middle surface; $\varepsilon_{1}$ and $\varepsilon_{2}$ - the relative elongation in the direction orthogonal dimensionless coordinates $\xi=x / R$ and $\theta$, where $x$-coordinate along the longitudinal axis of the shell; $\tau$ - twist of the middle surface along the shell; $u, v, w$ - related to the radius $R$ tangentially (along the coordinates $\xi$ and $\theta$ ) and radial movement of the middle surface of the shell.

$\rho_{n p}=\frac{\gamma_{c p}}{g}$ - the reduced density of the layers, $\gamma_{\mathrm{cp}}=\frac{\gamma_{1} h_{1}+\gamma_{2} h_{2}}{h}$ - averaged specific gravity of a double-layer shell, $h=h_{1}+h_{2}$ - thickness of the entire shell, $R_{0}$ - radius of the initial surface of a cylindrical shell. Let us turn to the solution of the problem in displacements that have basic relations with the semi-momentum shell theory:

$$
T_{1}=\left(1-v^{2}\right) B \varepsilon_{1}, M_{2}=D \chi_{2},
$$

where $E$ - the elastic modulus of the shell material; $\varepsilon_{1}$ - longitudinal deformation repose in two parts: $\varepsilon_{1}=\varepsilon_{0}+\frac{\partial u}{\partial \xi}, \varepsilon_{0}=\frac{F}{E A}, B=\frac{1}{1-v^{2}}\left(E_{1} h_{1}+E_{2} h_{2}\right)$, - the reduced rigidity of a doublelayer shell for tension (compression), $D=\frac{1}{3\left(1-v^{2}\right)}\left\{E_{1}\left[\left(h_{1}-z_{0}\right)^{3}+z_{0}^{3}\right]+E_{2}\left[\left(h_{2}+z_{0}\right)^{3}-z_{0}^{3}\right]\right\}$ - the 
reduced rigidity of a double-layer shell for bending. [8], $z_{0}=\frac{E_{1} h_{1}^{2}-E_{2} h_{2}^{2}}{2\left(E_{1} h_{1}+E_{2} h_{2}\right)}$, - position of the neutral surface of the shell.

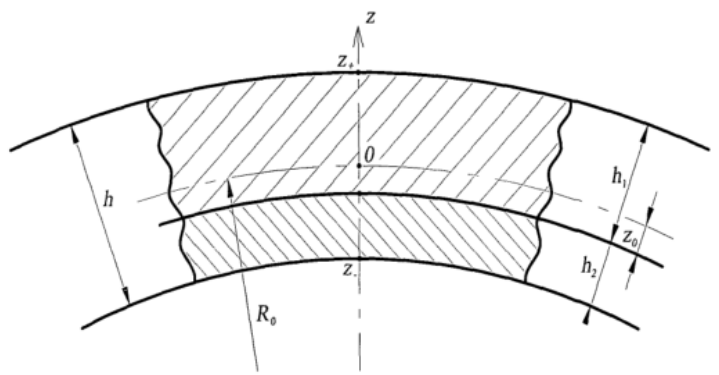

Fig. 2. Determination of the neutral surface of the shell

The initial deformation $\varepsilon_{0}$ determined on the assumption of non-deformable crosssections of the shell, $A$ - cross sectional area. the form:

The relations between deformations and displacements in semi-momentum theory have

$$
\frac{\partial v}{\partial \theta}+w=0 ; \quad \frac{\partial v}{\partial \xi}+\frac{\partial u}{\partial \theta}=0 ; \quad \vartheta_{2}=\frac{\partial w}{\partial \theta}-v ; \tau=-\frac{1}{R_{0}} \frac{\partial \vartheta_{2}}{\partial \xi}, \chi_{2}=-\frac{1}{R_{0}} \frac{\partial \vartheta_{2}}{\partial \theta}
$$

Substituting the relations (2), (3) into the equation (1) after some transformations and discarding the nonlinear terms, we get the linearized equation of shell in displacements:

$$
\begin{aligned}
\frac{\partial^{3} u}{\partial \xi^{3}}+\eta h_{v}^{2} \frac{\partial^{3}}{\partial \theta^{3}}\left(\vartheta_{2}+\frac{\partial^{2} \vartheta_{2}}{\partial \theta^{2}}\right)+ & 2 \frac{\partial^{2}}{\partial \theta^{2}}\left(\frac{\partial^{2} w}{\partial \xi^{2}} \varepsilon_{0}\right)-\frac{R_{0}}{E_{0} h} p_{0} \frac{\partial^{3} \vartheta_{2}}{\partial \theta^{3}}+\frac{R_{0}}{E_{0} h} \frac{\partial^{2}}{\partial \theta^{2}}[\Psi(\theta) w(\xi, \theta, t)]- \\
& -\frac{R_{0}^{2} \rho}{E_{0}}\left(\frac{\partial^{3} u}{\partial \xi \partial t^{2}}-\frac{\partial^{2} v}{\partial \theta \partial t^{2}}-\frac{\partial^{4} w}{\partial \theta^{2} \partial t^{2}}\right)=0
\end{aligned}
$$

where $u, v, w$ - components of the movement of the middle surface of the shell, divided by the radius R; $h_{v}$ - parameter of the relative thickness of the shell: $h_{v}=\frac{h}{R_{0} \sqrt{12\left(1-v^{2}\right)}}$, where $v$ - Poisson's ratio

$\Psi(\theta)=\frac{k R}{\pi}\left[\frac{\varphi_{0}^{3}}{3}+\sum_{m=1}^{\infty} \beta_{m} \cos m \theta\right] ; \quad \beta_{m}=(-1)^{m} m^{-3}\left[2 \varphi_{m} \cos \varphi_{m}-\left(\varphi_{m}^{2}-2\right) \sin \varphi_{m}\right] ; \quad \varphi_{m}=m \varphi_{0}$. $m=1,2,3 \ldots, \eta=\frac{E_{v}}{E_{0}}, E_{v}=\frac{\left(1-v^{2}\right) D}{h^{3}},-$ reduced modulus of elasticity of a double-layer shell for bending, $E_{0}=\frac{\left(1-v^{2}\right) B}{h},-$ reduced modulus of elasticity of a double-layer shell for tension (compression).

The obtained system of equations (4) contains four unknown functions of coordinates and time $\mathrm{t}: u, v, w$ and $\vartheta_{2}$. Solving this system by the method of separation of variables 
(Fourier method), we represent the function $w(\xi, \theta, t)$ in the case of hinged support end of shell, obeying the condition of periodicity on the circumferential coordinate $\theta$ :

$$
w=\sum_{m} \sum_{n} f(t) \sin \left(\tilde{\lambda}_{n} \xi\right) \cos (m \theta),
$$

where $\tilde{\lambda}_{n}=\frac{n \pi R}{L} ; m, n=1,2 \ldots$ - wavenumbers in the circumferential and longitudinal directions. The other components of displacement and rotation angle of the tangent determined from the relations of semi-momentum shell theory (3):

$$
\begin{aligned}
& u=-\sum_{m} \sum_{n} \frac{\tilde{\lambda}_{n}}{m^{2}} f(t) \cos \left(\lambda_{n} \xi\right) \cos (m \theta), \quad v=-\sum_{m} \sum_{n} \frac{1}{m} f(t) \sin \left(\tilde{\lambda}_{n} \xi\right) \sin (m \theta), \\
& \vartheta_{2}=-\sum_{m} \sum_{n} \frac{m^{2}-1}{m} f(t) \sin \left(\tilde{\lambda}_{n} \xi\right) \sin (m \theta) .
\end{aligned}
$$

Assuming that free vibrations of a shell is harmonic, we have:

$$
f(t)=\sin \omega_{m n} t, \quad f^{\prime \prime}(t)=-\omega_{m n}^{2} \sin \omega_{m n} t,
$$

where $\omega_{m n}-$ circular frequency of the shell on the form of the free bending vibrations $m, n=1,2,3 \ldots$.

Substituting (5) - (6) into the equation (4) and calculating the derivatives entering into the equation:

$$
\begin{aligned}
\frac{\partial^{3} u}{\partial \xi^{3}} & =-\sum_{m} \sum_{n} \frac{\lambda_{n}^{4}}{m^{2}} f(t) \sin \lambda_{n} \xi \cos m \theta \\
\frac{\partial^{5} \vartheta_{2}}{\partial \theta^{5}} & =\sum_{m} \sum_{n} m^{4}\left(m^{2}-1\right) f(t) \sin \lambda_{n} \xi \cos m \theta \\
\frac{\partial^{3} \vartheta_{2}}{\partial \theta^{3}} & =-\sum_{m} \sum_{n} m^{2}\left(m^{2}-1\right) f(t) \sin \lambda_{n} \xi \cos m \theta \\
\frac{\partial^{3} u}{\partial \xi \partial t^{2}} & =\sum_{m} \sum_{n} \frac{\lambda_{n}^{2}}{m^{2}} f^{\prime \prime}(t) \sin \lambda_{n} \xi \cos m \theta \\
\frac{\partial^{3} v}{\partial \theta \partial t^{2}} & =-\sum_{m} \sum_{n} f^{\prime \prime}(t) \sin \lambda_{n} \xi \cos m \theta \\
\frac{\partial^{4} w}{\partial \theta^{2} \partial \xi^{2}} & =\sum_{m} \sum_{n} m^{2} \lambda_{n}^{2} f(t) \sin \lambda_{n} \xi \cos m \theta \\
\frac{\partial^{4} w}{\partial \theta^{2} \partial t^{2}} & =-\sum_{m} \sum_{n} m^{2} f^{\prime \prime}(t) \sin \lambda_{n} \xi \cos m \theta
\end{aligned}
$$

We represent the result of differentiation of the term $\frac{\partial^{2}}{\partial \theta^{2}}[\Psi(\theta) w(t, \xi \theta)]$ as a product of partial sums. First, we multiply the series, and then compute the derivative by general rules. We introduce two partial sums: 


$$
\begin{aligned}
& S_{1}=\sum_{m} a_{m} \cos (m \theta) ; \quad m=1,2,3, \ldots \quad a_{0}=0 . \\
& S_{2}=b_{0}+\sum_{m} b_{m} \cos (m \theta) ; \quad b_{0}=\frac{\varphi_{0}^{3}}{3} ; \quad b_{m}=\beta_{m} .
\end{aligned}
$$

Next we obtain:

$Z=S_{1} S_{2}=z_{0}+\sum_{k=1}^{\hat{m}} z_{k} \cos k \theta$

The differentiation of the sum reduces to the differentiation of the terms:

$\frac{\partial}{\partial \theta} Z=-\sum_{k=1}^{\hat{m}} z_{k} k \sin k \theta . \quad \frac{\partial^{2}}{\partial \theta^{2}} Z=-\sum_{k=1}^{\hat{m}} z_{k} k^{2} \cos k \theta$.

For further calculations, we pass to the notation $k=m$. As a result, we obtain:

$$
\frac{R}{E h} \frac{\partial^{2}}{\partial \theta^{2}}\left(\Psi(\theta) w(\xi, \theta, t)=\frac{k R^{2}}{\pi E h} f(t) \sum_{m=1}^{\infty} m^{2} z_{m} \cos m \theta,\right.
$$

where $\varphi_{0}$ is given by (2.8), $\beta_{m}=(-1)^{m} m^{-3}\left[2 \varphi_{m} \cos \varphi_{m}-\left(\varphi_{m}^{2}-2\right) \sin \varphi_{m}\right]$,

$\varphi_{m}=m \varphi_{0}, k-$ coefficient of soil $\left[\mathrm{N} / \mathrm{m}^{3}\right], m-$ circumferential wave number.

Next, equating the coefficients of trigonometric functions $\cos m \theta$ at $m=1,2,3 \ldots$, we obtain an infinite system of homogeneous linear algebraic equations for the unknown amplitude values $b_{m}$ of radial displacement component $\mathrm{w}$.

$$
\begin{aligned}
& \left\{\widetilde{\lambda}_{n}^{4}+\eta m^{4}\left(m^{2}-1\right)\left(m^{2}-1+\frac{p^{*}}{\eta}\right)+k * m^{4}-2 \varepsilon_{0}^{*} \widetilde{\lambda}_{n}^{2} m^{4}\right\} f(t)+ \\
& +\rho_{n p}^{*} R_{0} h\left[\widetilde{\lambda}_{n}^{2} h_{v}+m^{2}+m^{4}\right] f^{\prime \prime}(t)=0,
\end{aligned}
$$

where $\tilde{\lambda}_{n}=\frac{n \pi R_{0}}{L \sqrt{h_{\widetilde{v}}}}, p^{*}=p \frac{R_{0}}{E_{0} h h_{v}^{2}}, \rho^{*}=\rho_{n p} \frac{R_{0}}{E_{0} h h_{v}^{2}}, \quad \varepsilon_{0}^{*}=\frac{F}{E_{0} A h_{v}{ }^{2}}, \quad k^{*}=\frac{R_{0}^{2} k}{\pi E_{0} h h_{v}^{2}} z_{m}$.

adding (7) to (10), we obtain:

$$
\tilde{\lambda}_{n}^{4}+\eta m^{4}\left(m^{2}-1\right)\left(m^{2}-1+\frac{p^{*}}{\eta}\right)+k^{*} m^{4}-2 \varepsilon_{0}^{*} \widetilde{\lambda}_{n}^{2} m^{4}-\rho_{n p}^{*} R_{0} \cdot h\left(\tilde{\lambda}_{n}^{2} h_{v}+m^{2}+m^{4}\right) \omega_{m n}^{2}=0
$$

We transform the term $2 \varepsilon_{0}^{*} \widetilde{\lambda}_{n}^{2} m^{4}$, taking into account the values of the cross-sectional area of a thin-walled tube $A=2 \pi R h$ and the axial moment of inertia $I=\pi R^{3} h$. As a result of transformations, we obtain:

$$
\begin{aligned}
& \tilde{\lambda}_{n}^{4}+m^{4}\left(m^{2}-1\right)\left(m^{2}-1+p^{*}\right)+k^{*} m^{4}-\widetilde{\lambda}_{n}^{4} m^{4} P / n^{2}- \\
& -\rho^{*} R \cdot h\left(\tilde{\lambda}_{n}^{2} h_{v}+m^{2}+m^{4}\right) \omega_{m n}^{2}=0,
\end{aligned}
$$

where $P=F / F_{\ni}$.

Transforming (11), we obtain the general expression for the square of the frequency of free oscillations $\omega_{m n}^{2}$ of rectilinear section of the pipeline (12). It allows us to study the influence of internal working pressure, the parameter of the longitudinal compressive force, 
geometrical characteristics, and the effect of the elastic foundation of the soil on the frequencies of free oscillations of a bimetallic surface pipeline of finite length $L$ :

$$
\omega_{m n}^{2}=\frac{\tilde{\lambda}_{n}^{4}+\eta m^{4}\left(m^{2}-1\right)\left(m^{2}-1+\frac{p^{*}}{\eta}\right)+k^{*} m^{4}-\tilde{\lambda}_{n}^{4} m^{4} P / n^{2}}{\rho_{n p}^{*} R_{0} \cdot h\left(\tilde{\lambda}_{n}^{2} h_{\tilde{v}}+m^{2}+m^{4}\right)},
$$

where $P=\frac{F}{F_{\ni}}-$ dimensionless parameter of the longitudinal compressive force, $F_{\ni}=\frac{\pi^{2} E I}{L^{2}}$ - Euler force, $\tilde{\lambda}_{n}=\frac{n \pi R_{0}}{L \sqrt{h_{v}}}$

If we equate the frequency to zero, we express the parameter of the internal working pressure:

$$
p^{*}=\frac{-\lambda_{n}^{4}}{m^{4}\left(m^{2}-1\right)}+\frac{\lambda_{n}^{4} P / n^{2}-k^{*}}{m^{4}\left(m^{2}-1\right)}-\eta\left(m^{2}-1\right)
$$

The obtained expression makes it possible to determine the critical pressure parameter at which the frequency of free oscillations is zero and the dynamic stability of the pipeline.

\section{Conclusions}

1. Based on the geometrically nonlinear variant of semi-momentum theory of cylindrical shells, the problem of determining the frequency of free oscillations of a thin-walled bimetallic pipeline of large diameter, which interacts with the elastic foundation, is solved. The solution takes into account the impact of the internal operating pressure, the geometric characteristics of the parameter of the longitudinal compressive force, and the influence of elastic resistance on the ground.

2. The obtained solution makes it possible to study the influence of materials of a doublelayer shell on the frequencies of free oscillations depending on their density, moduli of elasticity, and thickness.

3. It was found that the coefficient $\eta$ increases or decreases the critical value of the parameter of the internal operating pressure $p^{*}$, which indicates a change in the rigidity of the shell material in the longitudinal and circumferential directions.

The corresponding results in this paper were obtained during the execution of the state task of the Ministry of Education and Science of Russia 7.4794.2017/8.9.

\section{References}

1. Patent RU №2012155948/02, 21.12.2012. Safyanov AV, Fedorov AA

2. A.I. Zajcev, I.G.Radionova, A.V. Amežnov, et.al. Tehn. Kolesn I gus mashin. 3 (2013)

3. A.L. Bikbulatov, R.R. Bagmanov, K.M. Gumerov, Problemi sbora neft. Productov, 2(2), 120-126 (2015)

4. Li F., Wei B., Zhao X., Shao X., Cai R. Trenchless Technology - The Best Choice for Underground Pipeline Construction and Renewal, Proceedings of the International Conference on Pipelines and Trenchless Technology, (2013)

5. E.L. Akselrad, V.P. Il'in, Raschet truboprovodov. (Leningrad, Mashinostroenie, 1972)

6. V.G. Sokolov, I.O. Razov, Vest. grazhd. Inzh. 6 (2016)

7. V.G. Sokolov, PhD Thesis (Spb., 2011)

8. V.G. Sokolov, Prom.grajd.stroit 8, 34-48 (2010) 